\title{
Ground-state coordination of a catalytic metal to the scissile phosphate of a tertiary-stabilized Hammerhead ribozyme
}

\author{
W. LUKE WARD ${ }^{1,2}$ and VICTORIA J. DEROSE ${ }^{1,2,3}$ \\ ${ }^{1}$ Department of Chemistry, University of Oregon, Eugene, Oregon 97403-1253, USA \\ ${ }^{2}$ Institute of Molecular Biology, University of Oregon, Eugene, Oregon 97403-1253, USA
}

\begin{abstract}
Although the Hammerhead ribozyme (HHRz) has long been used as a model system in the field of ribozyme enzymology, several details of its mechanism are still not well understood. In particular, significant questions remain concerning the disposition and role of catalytic metals in the HHRz. Previous metal-rescue experiments using a "minimal" HHRz resulted in prediction of a catalytic metal that is bound in the A9/G10.1 site in the ground state of the reaction and that bridges to the scissile phosphate further along the reaction pathway. "Native" or extended HHRz constructs contain tertiary contacts that stabilize a more compact structure at moderate ionic strength. We performed $\mathrm{Cd}^{2+}$ rescue experiments on an extended $\mathrm{HHRz}$ from Schistosoma mansoni using stereo-pure scissile phosphorothioate-substituted substrates in order to determine whether a metal ion makes contact with the scissile phosphate in the ground state or further along the reaction coordinate. Inhibition in $\mathrm{Ca}^{2+} / \mathrm{Mg}^{2+}$ and rescue by thiophilic $\mathrm{Cd}^{2+}$ was specific for the $\mathrm{R}_{\mathrm{p}}-\mathrm{S}$ stereoisomer of the scissile phosphate. The affinity of the rescuing $\mathrm{Cd}^{2+}$, measured in two different ionic strength backgrounds, increased fourfold to 17-fold when the pro- $\mathrm{R}_{\mathrm{p}}$ oxygen is replaced by sulfur. These data support a model in which the rescuing metal ion makes a ground-state interaction with the scissile phosphate in the native HHRz. The resulting model for $\mathrm{Mg}^{2+}$ activation in the HHRz places a metal ion in contact with the scissile phosphate, where it may provide ground-state electrostatic activation of the substrate.
\end{abstract}

Keywords: Hammerhead ribozyme; metal rescue; catalytic RNA; kinetics; mechanism; metals

\section{INTRODUCTION}

A continuing challenge in the field of ribozyme enzymology is the precise determination of the contributions of metal ions to RNA catalytic activity. RNA has a general electrostatic requirement for cations for proper folding, and metal ions can specifically bind RNA ligands via inner or outer sphere coordination to provide a deeper level of structural stabilization. Site-specifically bound divalent metal ions can have a direct role in the chemical mechanism of the ribozyme as well (DeRose 2003; Sigel and Pyle 2007). Using RNase P and the group I intron as model ribozymes, Steitz and Steitz (1993) proposed that ribozymes might rely on bound metal ions to perform general acid/base chemistry. However, insights into the mechanisms of small nucleolytic ribozymes have indicated that the nucleobase functional

\footnotetext{
${ }^{3}$ Corresponding author.

E-mail derose@uoregon.edu.

Article published online ahead of print. Article and publication date are at http://www.rnajournal.org/cgi/doi/10.1261/rna.030239.111.
}

groups of RNA can also play critical roles in catalysis, with the extreme example of the hairpin ribozyme that is proposed to catalyze phosphoryl transfer in the complete absence of metal ion participation in the chemical mechanism (Nesbitt et al. 1997; Bevilacqua 2003). A thorough understanding of the range of mechanisms used by ribozymes has not yet been realized, because the detailed mechanisms including the specific roles of metal ions in ribozyme active sites are difficult to ascertain.

The Hammerhead ribozyme (HHRz) has been a particularly challenging system with regard to determining the roles of metal ions in structure and catalysis. The $\mathrm{HHRz}$ is a small nucleolytic ribozyme that catalyzes the site-specific nucleophilic attack of a $2^{\prime}-\mathrm{OH}$ on its $3^{\prime}$-phosphodiester bond, resulting in strand cleavage. Hammerhead ribozymes were originally discovered as the genome processing component of RNA-based viroids and have since been found in genomes throughout the domains of life (Buzayan et al. 1986; de la Pena and Garcia-Robles 2010). In medium ionic strength, HHRz activity is extremely sensitive to the concentration and identity of divalent metals (Hunsicker 
and DeRose 2000; Boots et al. 2008). Moderate rates of catalysis can also be achieved in molar concentrations of monovalent cations, an important property that helped to uncover the critical roles of nucleobases in the HHRz reaction mechanism (Murray et al. 1998; O’Rear et al. 2001; Bevilacqua et al. 2004). At physiological ionic strengths, the HHRz requires divalent ions for appreciable rates of catalysis; therefore, it is reasonable to assume that the divalent metal-dependent channel is the primary mode of catalysis in nature (Khvorova et al. 2003).

The HHRz was studied for years in its simplest active form, as three short helices meeting at a junction of conserved nucleotides that form the active site of the ribozyme (for review, see Blount and Uhlenbeck 2005). Studies using this "truncated" form of the HHRz (trHHRz) led to a model of catalysis in which a catalytic metal in the P9/ G10.1 site coordinates the pro-R oxygen of the scissile phosphate, presumably to stabilize the negative charge of the phosphorane transition state (Peracchi et al. 1997; Wang et al. 1999). Based on detailed metal-rescue experiments, Wang et al. (1999) predicted that the metal ion coordinates to the P9/G10.1 site in the ground state and bridges to the scissile phosphate in the transition state of the trHHRz reaction. A ground state that is very different from the transition state is consistent with structural studies of the truncated HHRz, which in general did not show catalytically relevant atoms within appropriate distances of the active site (Blount and Uhlenbeck 2005). In these structures, the P9/G10.1 metal ion site is $\sim 20 \AA$ away from its predicted ligand during catalysis, the pro-R oxygen of the scissile phosphate (Pley et al. 1994; Scott et al. 1995). To corroborate these seemingly conflicting data, it was proposed that the trHHRz periodically sampled a thermodynamically unstable "active" structure in which the implicated functional nucleobases and metal ion would be in the correct position to stimulate catalysis (for review, see Blount and Uhlenbeck 2005).

Tertiary-stabilized or "extended" Hammerhead ribozymes contain nonconserved unpaired regions in helices I and II that interact to promote the formation of the active site in the helix junction. These tertiary interactions result in a higher population of functionally folded ribozymes, leading to higher observed rates at significantly lower divalent metal concentrations in vitro, as well as activity in vivo (Khvorova et al. 2003). New structures of constructs based on native HHRz's (2GOZ, 2OEU, 2QUS) largely satisfy predictions based on previous mutations and also provide new hypotheses concerning players in the HHRz mechanism (Martick and Scott 2006; Lee et al. 2007; Chi et al. 2008; Martick et al. 2008). The conformation of the native HHRz in crystalforming conditions is significantly different from that of the truncated HHRz. The native HHRz structures show a wellorganized active site, with functional groups from the G8 and G12 guanosines in place to participate in general acid/ base catalysis (Fig. 1). One fascinating aspect of these

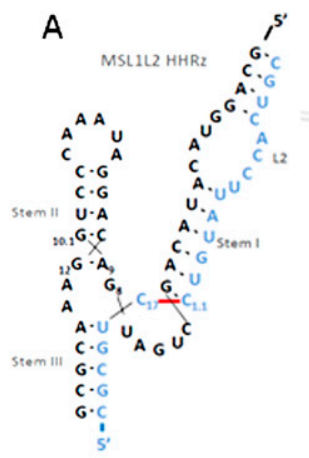

C
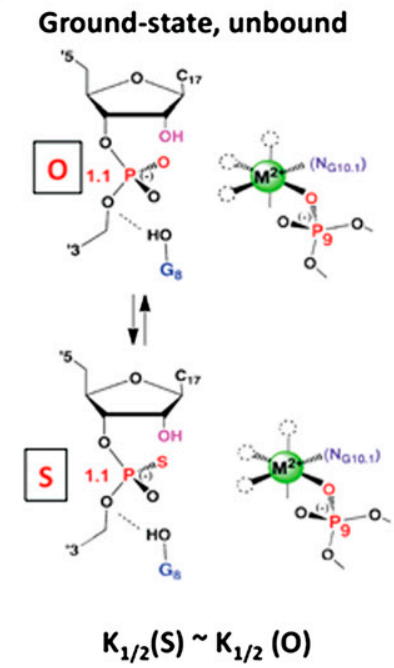

B

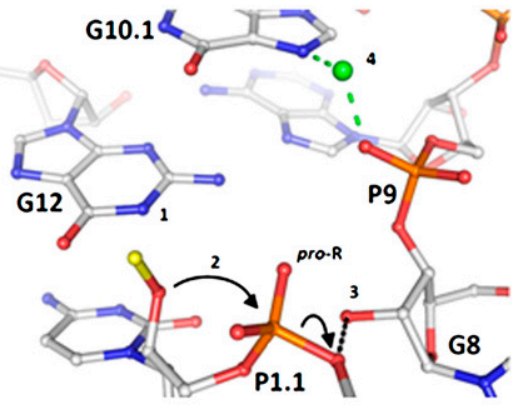

Ground-state, bound

's
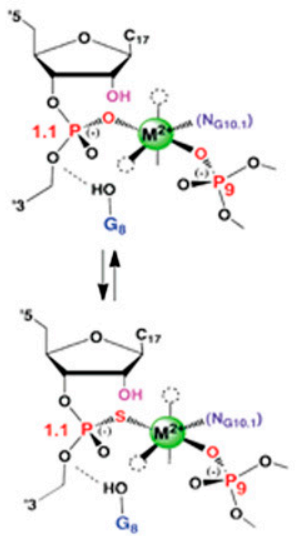

$K_{1 / 2}(S)<K_{1 / 2}(0)$
FIGURE 1. (A) Secondary structure of the modified Schistosoma mansoni HHRz (MSL1L2) (Osborne et al. 2005) used in these studies. (B) Crystallographic active site of the S. mansoni HHRz (2OEU) (Martick et al. 2008) showing (1) proximity of the G12 N1, proposed to act as general base for activation of the $2^{\prime}$ nucleophile $\left(2\right.$, a $2^{\prime}$-OMe in this structure) and the $2^{\prime}-\mathrm{OH}$ of G8 that has been proposed to act as a general acid for the $5^{\prime}-\mathrm{O}^{-}$leaving group (3). The $\mathrm{Mn}^{2+}$ ion positioned in the P9/G10.1 active site (4) would be proposed to coordinate the pro$\mathrm{R}$ oxygen of the scissile phosphate based on these studies. $(C)$ Results expected for $\mathrm{Cd}^{2+}$ rescue of phosphorothioate substitution at the $\mathrm{HHRz}$ scissile phosphate. If there is no ground-state interaction between the metal and the substituted site (left), the value of $K_{1 / 2}\left(\mathrm{Cd}^{2+}\right)$ for $\mathrm{Cd}^{2+}$ activation of the reaction should not change for the $\mathrm{O}$ and $\mathrm{S}$ substrates. For a ground-state interaction, $K_{1 / 2}\left(\mathrm{Cd}^{2+}\right)$ should decrease to reflect the higher affinity of the $\mathrm{Cd}^{2+}-\mathrm{S}$ interaction in the substituted site.

structures, however, is that even in this proposed "active" conformation, the metal modeled at the P9/G10.1 site is still not making contact with functional groups directly involved in catalysis.

The extended HHRz from Schistosoma mansoni demonstrates $\mathrm{Cd}^{2+}$ rescue of P9 and scissile phosphorothioate substitutions, supporting the prediction that inner-sphere coordination of a metal ion to these phosphates is required for efficient catalysis in the stabilized HHRz (Osborne et al. 2005). These prior studies were done with mixtures of phosphorothioates and fixed $\mathrm{Cd}^{2+}$ concentrations and did not provide sufficient basis to determine ground-state affinities of the rescuing metal ions. Assuming that the metal 
ion observed in the P9/G10.1 site of native HHRz's is the catalytically relevant ion implicated in coordinating the pro- $\mathrm{R}$ oxygen of the scissile phosphate, as is predicted by studies in the trHHRz, two simple models exist that might explain how this metal ion interacts with the active site during catalysis. In one model, named here the "dynamic model," the metal ion could be recruited to the scissile phosphate at some point during the reaction resulting in stabilization of the negative charge of the phosphorane transition state (Wang et al. 1999). This model, combined with native $\mathrm{HHRz}$ crystal structures, has been the basis for recent computational studies that calculated the position of the metal ion during different points along the $\mathrm{HHRz}$ reaction coordinate. These molecular simulations began with the crystallographically predicted P9/G10.1 metal site and predicted that a slight negative charge build-up resulting from nucleophile deprotonation is enough to rapidly and stably recruit the P9/G10.1 metal into a bridging coordination mode between the pro-R oxygen atoms of the P9 and scissile phosphates (Lee et al. 2008, 2009). Alternatively, the positioning of the metal ion may be specific to crystallography, in that the described structure is the most stable state under crystal-forming conditions. In solution, the most active form of the ribozyme may be slightly different or be in equilibrium with the conformation observed in X-ray structures. A pre-initiation coordination (or an "activated ground-state coordination") of the catalytically relevant metal ion to the scissile phosphate could theoretically provide a strong chemical advantage over the dynamic model via several methods. For instance, in a ground-state pro-R oxygen coordination position, the metal ion could lower the reaction barrier by stabilizing in-line attack geometry, activating the nucleophile via direct or indirect coordination, and/or withdrawing electron density from the phosphate, thereby increasing the electrophilicity of the scissile phosphorus. Proximity of the metal ion may also lower the $\mathrm{p} K_{\mathrm{a}}$ of the putative general base and/ or nucleophilic $2^{\prime}-\mathrm{OH}$. In the predicted mechanisms of other metal-dependent ribozymes such as the Group I intron, one or more metal ions are coordinated in the active site of the ribozyme without the need for metal recruitment during the reaction (Frederiksen and Piccirilli 2009).

We have performed detailed cadmium rescue experiments on HPLC-separated stereoisomers of a scissile phosphorothioate-substituted $\mathrm{HHRz}$ derived from S. mansoni in order to test whether a catalytic metal coordinates the scissile phosphate in the ground state or is instead recruited to the scissile phosphate during the transition state of the HHRz mechanism. Our results are consistent with a mechanism in which the rescuing metal makes a ground-state coordination with the pro-R oxygen of the scissile phosphate. These results differ from prior studies of cadmium rescue using trHHRz constructs, which demonstrated transition-state and not ground-state coordination (Peracchi et al. 1997; Wang et al. 1999), indicating that the tertiary interactions in native $\mathrm{HHRz}$ constructs stabilize a ground state that may be more advanced on the reaction coordinate. These results also imply that the current structural view of the $\mathrm{HHRz}$, with a metal ion coordinated only in the P9/G10.1 site, may not represent the most active conformation of the $\mathrm{HHRz}$ active site. Instead, the crystal structure may represent just one of several conformations available to the $\mathrm{HHRz}$ core leading to catalytic activation, whereby the activating metal coordinates the pro-R oxygen of the scissile phosphate to stimulate catalysis.

\section{RESULTS AND DISCUSSION}

\section{$\mathrm{Cd}^{2+}$ rescue in $100 \mathrm{mM} \mathrm{Na}^{+}$ and $15 \mathrm{mM} \mathrm{Ca}^{2+}$}

The HHRz has a general dependence on divalent metal cations for folding. In a background of $100 \mathrm{mM} \mathrm{Na}^{+}$, the $K_{1 / 2}$ for folding is $\sim 1 \mathrm{mM} \mathrm{M}^{2+}$ for a variety of metal cations (Kim et al. 2005; Boots et al. 2008; WL Ward and VJ DeRose, in prep.). Catalytic activation of the ribozyme occurs at much higher metal concentrations than does folding, however, and reaction rates vary greatly depending on cation identity (Kim et al. 2005; Boots et al. 2008; WL Ward and VJ DeRose, in prep.). For instance, $\mathrm{Cd}^{2+}$ is an especially stimulatory cation, while $\mathrm{Ca}^{2+}$ is a poor stimulator of activity (Boots et al. 2008). We used these relationships in thiophosphate substitution- $\mathrm{Cd}^{2+}$ rescue experiments to separate the effects of $\mathrm{Cd}^{2+}$ on catalytic rescue from its contributions to global folding. HHRz kinetic experiments were performed in a background of $15 \mathrm{mM} \mathrm{Ca}^{2+}$ and $100 \mathrm{mM}$ $\mathrm{Na}^{+}$, which supports a folded ribozyme population with very low activity, and the ability of $\mathrm{Cd}^{2+}$ to stimulate wild-type (WT) and thiophosphate-substituted samples was compared.

Stereosiomers $\left(R_{p}\right.$ and $\left.S_{p}\right)$ of the scissile phosphorothioate-substituted $\mathrm{HHRz}$ substrate strand were separated by reverse-phase HPLC for use in $\mathrm{Cd}^{2+}$ rescue titrations alongside the wild-type scissile phosphate construct (WT). The rate for each construct in $15 \mathrm{mM} \mathrm{Ca}^{2+}$ and $100 \mathrm{mM}$ $\mathrm{Na}^{+}$is very low, reaching only $0.04 \mathrm{~min}^{-1}$ for the WT construct (Fig. 2). The $S_{p}$ construct has a rate reduction of one-half the WT rate $\left(k=0.02 \mathrm{~min}^{-1}\right)$, indicating that the $\mathrm{S}_{\mathrm{p}}$ construct is only slightly inhibited by the sulfur substitution. In contrast, the $\mathrm{R}_{\mathrm{p}}$ construct cleaves 100 -fold more slowly than the WT construct under these conditions $\left(k=0.0004 \mathrm{~min}^{-1}\right)$. General catalytic inhibition by a phosphorothioate substitution is possible in ribozymes (Forconi and Herschlag 2009) and could be due to slight structural perturbation caused by the larger sulfur atom in place of an oxygen atom. Additionally, the less electronegative sulfur atom may withdraw less electron density from the phosphorus, causing the phosphorus to have a lower electrophilicity than the native phosphate, leading to a lower intrinsic reaction rate (Erat and Roland 2011). The 50 -fold additional inhibition from the $\mathrm{R}_{\mathrm{p}}$ thio substitution 


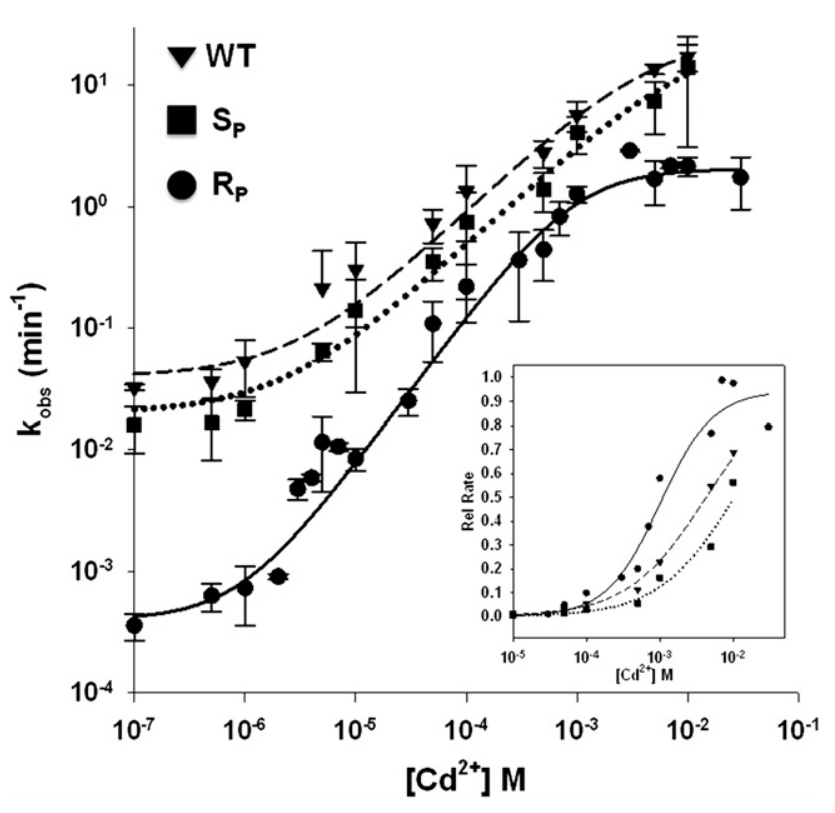

FIGURE 2. Cadmium rescue of HHRz constructs in $100 \mathrm{mM} \mathrm{Na}^{+}$ and $15 \mathrm{mM} \mathrm{Ca}^{2+}$. The wild-type (WT) (triangle) and $S_{\mathrm{p}}$ phosphorothioate construct (square) show similar $\mathrm{Cd}^{2+}$ profiles compared with the $R_{p}$ phosphorothioate construct (circle), which is significantly inhibited in the absence of $\mathrm{Cd}^{2+}$. The rates in $0 \mathrm{mM} \mathrm{Cd}^{2+}$ are 0.04 $\mathrm{min}^{-1}$ for WT, $0.02 \mathrm{~min}^{-1}$ for $S_{\mathrm{P}}$ construct, and $0.0004 \mathrm{~min}^{-1}$ for the $\mathrm{R}_{\mathrm{p}}$ construct. The data were fit to Equation 3 to give apparent binding affinities of the rescuing $\mathrm{Cd}^{2+}$ of WT $K_{1 / 2}=4 \pm 1 \mathrm{mM}, \mathrm{S}_{\mathrm{p}} K_{1 / 2}=10 \pm$ $7 \mathrm{mM}$, and $\mathrm{R}_{\mathrm{p}} K_{1 / 2}=900 \pm 300 \mu \mathrm{M}$. (Inset) Presents the data using rates normalized to the predicted $k_{\max }$ in order to highlight the differences in $K_{1 / 2}$ values.

in comparison with the $S_{p}$ isomer is consistent with further loss of activity that may be due to the very low affinity of the hard $\mathrm{Ca}^{2+}$ ion for the $\mathrm{R}_{\mathrm{p}}$ sulfur.

The addition of $\mathrm{Cd}^{2+}$, which supports faster reaction rates in comparison with both $\mathrm{Mg}^{2+}$ and $\mathrm{Ca}^{2+}$, causes a rate increase for each $\mathrm{HHRz}$ construct. Upon titration with $\mathrm{Cd}^{2+}$, the activity of the $\mathrm{S}_{\mathrm{p}}$ construct approximately mirrors that of the WT, with the $S_{p}$ phosphorothioate causing a small and constant rate reduction (Fig. 2). Due to the limits of manual kinetics techniques, rates for the $S_{p}$ and WT ribozyme were not collected above $10 \mathrm{mM} \mathrm{Cd}^{2+}$. The data were fit to Equation 3 (in Materials and Methods) and give a predicted $k_{\max }$ of $\sim 25 \mathrm{~min}^{-1}$ for both ribozymes, which likely represents a lower limit for the $k_{\max }$ values. The stimulation of WT activity over this range of $\mathrm{Cd}^{2+}$ concentrations is $\sim 600$-fold. Over the same range of $\mathrm{Cd}^{2+}$ concentrations, the initial inhibition in the $R_{p}$ construct is almost completely rescued, ending in a saturated activity curve with a $k_{\max }$ that is $\sim 10$-fold lower than the WT ribozyme. Incomplete rescue of the $\mathrm{R}_{\mathrm{p}}$ construct to the WT rates may be due to the properties of phosphorothioate substitutions mentioned above. The 5000-fold stimulation in $R_{p}$ activity, however, is consistent with the rescue of an essential metal coordination with the pro- $\mathrm{R}$ position of the scissile phosphate.
The $K_{1 / 2}$ value of activity stimulation can be considered to reflect the apparent affinity of the activating metal to the HHRz in the ground state. Therefore, if the coordination environment of the metal ion in the activated complex is changed such that the affinity between a metal ion and the respective ligand is increased, this should be reflected as a decrease in the $K_{1 / 2}$ of stimulation (Fig. 1C). The WT ribozyme represents the standard state of the metal binding pocket, with the "rescuing" or "stimulating" metal ion coordinating a given set of ligands. If the metal ion coordinates a scissile phosphate oxygen in the ground state, then in the thio-substituted construct the increased affinity of a $\mathrm{Cd}^{2+}-\mathrm{S}$ bond over a $\mathrm{Cd}^{2+}-\mathrm{O}$ bond should be reflected in an increased affinity of the rescuing metal. Based on the data of Figure 2, the WT and $S_{p}$ constructs have predicted stimulation $K_{1 / 2}$ values of $4 \pm 1 \mathrm{mM}$ and $10 \pm 7 \mathrm{mM}$, respectively, for $\mathrm{Cd}^{2+}$ stimulation in a background of $15 \mathrm{mM}$ $\mathrm{Ca}^{2+} / 100 \mathrm{mM} \mathrm{Na}{ }^{+}$. Due to incomplete saturation of $k_{\text {max }}$, these values likely represent lower limits for the apparent $K_{1 / 2}$ of the catalytically stimulating metal. For the $\mathrm{R}_{\mathrm{p}}$ construct, a $K_{1 / 2}=900 \pm 300 \mu \mathrm{M} \mathrm{Cd}^{2+}$ is measured, which is fourfold to 10 -fold lower than the constructs with a pro- $\mathrm{R}$ oxygen. This increased affinity of the rescuing metal for the $\mathrm{R}_{\mathrm{p}}$ thio-construct is consistent with coordination of the activating metal to the pro- $\mathrm{R}$ position of the scissile phosphate in the ground state of the HHRz.

In model nucleotide systems, the binding affinity of $\mathrm{Cd}^{2+}$ to a phosphorothioate is $20-100$-fold tighter than the affinity to a phosphate oxygen (Pecoraro et al. 1984; Sigel et al. 1997). The affinity increase in these model systems calls into question whether a fourfold to 10 -fold increase can correctly be considered to reflect a "ground-state" coordination. Consideration should be given to the fact that the rescuing metal ion in our experiment competes with $15 \mathrm{mM} \mathrm{Ca}^{2+}$ and $100 \mathrm{mM} \mathrm{Na}^{+}$for positioning in the active site. Wang et al. (1999), using similar ionic conditions of $10 \mathrm{mM} \mathrm{Ca}^{2+} / 100 \mathrm{mM} \mathrm{Na}^{+}$, measured a fourfold increase in apparent affinity for $\mathrm{Cd}^{2+}$ stimulation of activity in a trHHRz with an $A 9 \mathrm{R}_{\mathrm{p}}-\mathrm{S}$ substitution, leading to the conclusion that the rescuing metal ion made a ground-state interaction with the A9 pro- $\mathrm{R}$ oxygen. For the same P9 substitution but in a background of $10 \mathrm{mM} \mathrm{Mg}^{2+}$, Peracchi et al. (1997) found an 8.8-fold increased $K_{1 / 2}$ for stimulating $\mathrm{Cd}^{2+}$. Metal coordination to the $\mathrm{A} 9 / \mathrm{G} 10.1$ site in activity-inhibited constructs has also been spectroscopically measured, providing further support for the description of the A9 interaction as a ground-state interaction (Horton et al. 1998; Hunsicker and DeRose 2000; Suzumura et al. 2002; Vogt et al. 2006). Additionally, in the present study, the $K_{1 / 2}$ values for $\mathrm{Cd}^{2+}$ activity stimulation in the $\mathrm{WT}$ and $\mathrm{S}_{\mathrm{p}}$ constructs likely represent low estimates of the actual $K_{1 /}$ 2 values in this ionic background. Therefore, it is likely that the $K_{1 / 2}$ difference between the $\mathrm{R}_{\mathrm{p}}$ and the pro- $\mathrm{R}$ oxygen constructs is also a lower limit. The fourfold to 10 -fold increase in the affinity of $\mathrm{Cd}^{2+}$ for the $\mathrm{R}_{\mathrm{p}}$ scissile phos- 
phorothioate in the native $\mathrm{HHRz}$ is, therefore, consistent with affinity increases of other characterized ground-state interactions in the HHRz.

\section{$\mathrm{Cd}^{2+}$ rescue in $20 \mathrm{mM} \mathrm{Na}^{+}$and $5 \mathrm{mM} \mathrm{Ca}^{2+}$}

Metal coordination is subject to general cation competition, and therefore the presence of high concentrations of $\mathrm{Na}^{+}$and $\mathrm{Ca}^{2+}$, while intended to deconvolute the role of $\mathrm{Cd}^{2+}$ in metal rescue, will also raise the level of $\mathrm{Cd}^{2+}$ required for achieving maximum activities. We have recently used 2-aminopurine fluorescence to measure global $\mathrm{HHRz}$ folding in a reduced ionic background of $20 \mathrm{mM} \mathrm{Na}^{+}$and found a $K_{1 / 2, \text { fold }}{ }^{\mathrm{Ca}}$ value of $230 \pm 20 \mu \mathrm{M}$ (WL Ward and VJ DeRose, in prep.). The lowered $\mathrm{Na}^{+}$background also reduces the $k_{\max }$ and $K_{1 / 2}$ for $\mathrm{Mg}^{2+}$ activation. By reducing the background $\mathrm{Na}^{+}$and $\mathrm{Ca}^{2+}$ concentrations, competition for the rescuing $\mathrm{Cd}^{2+}$ ion should be reduced in the $\mathrm{HHRz}$ active site while still satisfying the general ionic requirements for global folding. We therefore repeated the $\mathrm{Cd}^{2+}$ activity titrations for the WT and $\mathrm{R}_{\mathrm{p}}$ constructs in a background of $20 \mathrm{mM} \mathrm{Na}^{+}$and $5 \mathrm{mM} \mathrm{Ca}^{2+}$ (Fig. 3).

Under these lower background ionic conditions, the $k_{\max }{ }^{\text {app }}$ for stimulating $\mathrm{Cd}^{2+}$ is reduced in each construct, consistent with observations for $\mathrm{Mg}^{2+}$ activation in reduced

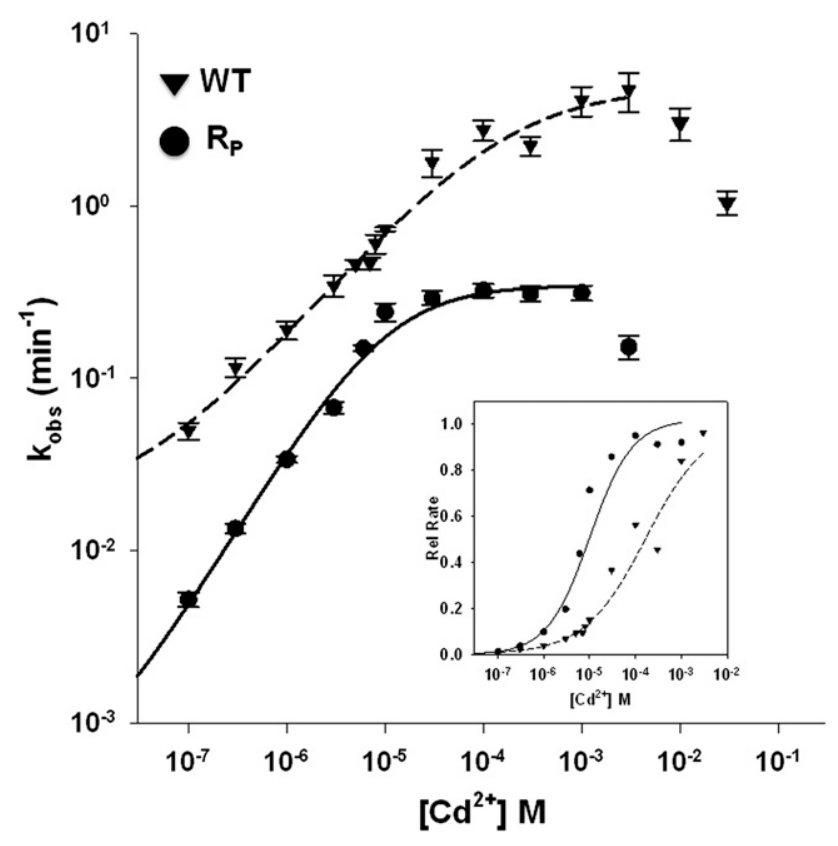

FIGURE 3. Cadmium rescue of the HHRz construct in $20 \mathrm{mM} \mathrm{Na}^{+}$ and $5 \mathrm{mM} \mathrm{Ca}^{2+}$. WT (triangle) and $\mathrm{R}_{\mathrm{p}}$ phosphorothioate construct (circle) both experience catalytic inhibition phases at $\left[\mathrm{Cd}^{2+}\right]>3 \mathrm{mM}$. The rates in $0 \mathrm{mM} \mathrm{Cd}^{2+}$ are $0.02 \mathrm{~min}^{-1}$ for WT and $<0.0004 \mathrm{~min}^{-1}$ for the $\mathrm{R}_{\mathrm{p}}$ construct. The data, excluding inhibited points, were fit to Equation 3 to give apparent binding affinities of the rescuing $\mathrm{Cd}^{2+}$ of WT $K_{1 / 2}=170 \pm 10 \mu \mathrm{M}$ and $\mathrm{R}_{\mathrm{p}} K_{1 / 2}=10 \pm 3 \mu \mathrm{M}$. (Inset) Presents the data using rates normalized to the predicted $k_{\max }$ in order to highlight the differences in $K_{1 / 2}$ values.
$\mathrm{Na}^{+}$(WL Ward and VJ DeRose, in prep.). With added $\mathrm{Cd}^{2+}$ the activity of the WT construct increases and then peaks at $\sim 3 \mathrm{mM} \mathrm{Cd}^{2+}$, followed by catalytic inhibition at higher concentrations of $\mathrm{Cd}^{2+}$. Inhibition could be explained by the presence of a relatively low-affinity metal coordination site that stabilizes an inactive conformer of the HHRz at high concentrations of $\mathrm{Cd}^{2+}$. Catalytic inhibition by soft metals is a known problem due to the propensity for non-native site binding (Forconi and Herschlag 2009), and $\mathrm{Cd}^{2+}$ inhibition of the trHHRz has been observed as well (Wang et al. 1999). The $R_{p}$ construct is able to reach a saturated $\mathrm{k}_{\max }$ at low micromolar $\mathrm{Cd}^{2+}$ concentrations, and it too begins to show catalytic inhibition at $3 \mathrm{mM} \mathrm{Cd}^{2+}$, supporting this inhibition as a general effect of high $\mathrm{Cd}^{2+}$ on the $\mathrm{HHRz}$ in these background ionic conditions. Since this inhibitory effect was not observed in $100 \mathrm{mM} \mathrm{Na}^{+}$and $15 \mathrm{mM} \mathrm{Ca}^{2+}$, it may indicate that the inhibitory metal is effectively outcompeted by higher background ionic conditions. Additionally, as it is also not observed for $\mathrm{Mg}^{2+}$ (Kim et al. 2005), this inhibitory effect may be specifically attributed to the binding of soft metal ions or transition metal ions.

The WT and $\mathrm{R}_{\mathrm{p}} \mathrm{Cd}^{2+}$ rate profiles, excluding the inhibition phase, were fit to Equation 3. The reduced cation competition clearly amplifies the differences in $K_{1 / 2}$ values for $\mathrm{Cd}^{2+}$ stimulation of activity in these constructs. In $5 \mathrm{mM} \mathrm{Ca}^{2+} / 20 \mathrm{mM} \mathrm{Na}^{+}$, the $K_{1 / 2}$ for $\mathrm{Cd}^{2+}$ stimulation of WT activity is $170 \pm 10 \mu \mathrm{M}$, while the $K_{1 / 2}$ for the $R_{p}$ construct is now $10 \pm 3 \mu \mathrm{M}$. This 17 -fold increase of $\mathrm{Cd}^{2+}$ affinity for the $\mathrm{R}_{\mathrm{p}}$ thio-construct over the WT phosphate strongly supports a ground-state metal coordination to the pro-R oxygen of the HHRz.

\section{CONCLUSIONS/SUMMARY}

The HHRz relies on divalent metal ions to achieve appreciable rates of catalysis in physiological ionic strength. We investigated the catalytically important coordination of a metal ion to the scissile phosphate using $\mathrm{Cd}^{2+}$ rescue titrations under ionic conditions in which the HHRz is globally folded with low background catalytic activity. Consistent with previous work on minimal $\mathrm{HHRz}$ constructs, we find that $\mathrm{Cd}^{2+}$ rescue is stereo-specific for the pro- $\mathrm{R}$ position of the scissile phosphate. However, we also find a fourfold to 17-fold increased affinity of the rescuing $\mathrm{Cd}^{2+}$ for the $\mathrm{R}_{\mathrm{p}}$ phosphorothioate construct. This increased affinity of the rescuing metal supports a model in which a catalytic metal coordinates the pro- $\mathrm{R}$ oxygen of the scissile phosphate in the ground state of the HHRz mechanism, a result that differs from current mechanistic models derived from trHHRz data in combination with crystallographic structures of native HHRz's (Wang et al. 1999; Lee et al. 2009).

The "minimal" trHHRz that lacks tertiary interactions is proposed to predominantly occupy an "open" state that requires a conformational change to a "closed" or active 
state for catalysis. This closed form seems to be only transiently populated, meaning that "ground-state" pre-equilibration of metal occupancies in the trHHRz would mainly reflect the open conformation. Both structure (Scott et al. 1995) and spectroscopic measurements (Maderia et al. 2000; Vogt et al. 2006; Osborne et al. 2009) indicate higheraffinity population of the P9/G10.1 metal ion site in the trHHRz. Thus, metal-rescue studies of the trHHRz that predict metal coordination to the $\mathrm{P} 9$ site in the ground state, and the scissile phosphate at a later point on the reaction pathway, are consistent for that system.

Native HHRz constructs, however, stabilize a "closed" conformation through tertiary interactions. Given the higher stability of this closed conformation and the fact that P9 and scissile phosphate oxygens are much closer in native $\mathrm{HHRz}$ crystal structures, a bridging coordination mode for the catalytic metal ion seems to be achievable in the ground state of these constructs. Despite this, current crystallographic models locate a $\mathrm{Mn}^{2+}$ ion only in the nearby P9/G10.1 coordination site (Martick et al. 2008) or not strongly localized in the case of $\mathrm{Mg}^{2+}$ (Martick and Scott 2006; Chi et al. 2008). In contrast, the data presented here strongly support a ground-state metal coordination to the scissile phosphate. A caveat to thio-substitution and $\mathrm{Cd}^{2+}$ rescue experiments is the potential creation of a nonnative coordination environment due to the increased affinity of $\mathrm{Cd}^{2+}$ for sulfur over oxygen. The potential drawbacks of metal-rescue methods for ribozyme mechanistic studies are well discussed in the literature (Forconi and Herschlag 2009; Frederiksen and Piccirilli 2009; Erat and Roland 2011). While caveats must be considered, such methods have been used successfully to identify important coordination sites for several ribozymes (Frederiksen and Piccirilli 2009). A quantitative analysis of the apparent affinity of the rescuing metal, such as performed here, expands the information gained to identify the point along the reaction coordinate at which the metal ion contacts the substituted ligand. The fourfold to 17-fold increase in affinity for $\mathrm{Cd}^{2+}$ for the substituted $\mathrm{R}_{\mathrm{p}}$ scissile phosphorothioate observed for this native $\mathrm{HHRz}$ is very similar to results previously obtained for the P9/ G10.1 site in the trHHRz, whose occupancy has been corroborated by crystallography and spectroscopic methods. The simplest interpretation of the current data is therefore that the HHRz has a catalytic metal ion bound to the scissile phosphate. Although current crystallographic results place a metal ion in the nearby P9/G10.1 position, simulations in which the metal has moved to bridge to the scissile phosphate show small structural changes, including an increase in the in-line fitness for nucleophilic attack (Lee et al. 2008). Structural changes along with beneficial electrostatic effects would enhance the catalytic potential of the ribozyme. We propose, therefore, that the most active form of the HHRz involves a ground-state coordination of an activating metal ion to the pro- $\mathrm{R}$ oxygen of the scissile phosphate, which is then expected to aid catalysis throughout the reaction coordinate.

\section{MATERIALS AND METHODS}

\section{Oligonucleotides}

RNA oligomers were purchased from Dharmacon (CO), deprotected according to the manufacturer's instructions, reconstituted in deionized and filtered water, and quantified by absorbance at $260 \mathrm{~nm}$. The sequences for ribozyme and substrate strands are as follows: Enzyme Strand: 5'-GCAGGUACAUACAGCUGAUGA GUCCCAAAUAGGACGAAACGCG. Substrate Strand: 5' -CGCG $\mathrm{UC}^{\star} \mathrm{CUGUAUUCCACUGC}$. The asterisk denotes the scissile phosphorothioate substitution site.

\section{Reagents}

All reagents used were molecular biology grade or better. $\mathrm{CaCl}_{2} \bullet 2$ $\mathrm{H}_{2} \mathrm{O}$ was obtained from Sigma-Aldrich and $\mathrm{Cd}\left(\mathrm{NO}_{3}\right)_{2} \bullet 4 \mathrm{H}_{2} \mathrm{O}$ from Alfa Aesar.

\section{Separation of phosphorothioate stereoisomers}

Phosphorothioate-substituted RNAs consisting of a mixture of the $\mathrm{S}_{\mathrm{p}}$ and $\mathrm{R}_{\mathrm{p}}$ diastereomers were separated using reverse-phase HPLC (Akta purifier; Amersham Biosciences) on a C18 column (Hypersil

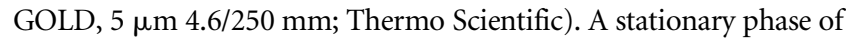
$10 \mathrm{mM}$ triethylammonium acetate (TEAA) and a mobile phase of $80 \%$ acetonitrile/20\% $10 \mathrm{mM}$ TEAA were used. A multistep gradient was used that provided baseline separation of two major peaks.

The configurations of the separated stereoisomers were determined using stereospecific digestion by phosphodiesterase I (Slim and Gait 1991). This treatment leaves an undigested dinucleotide in the $S_{p}$ fraction that is identified using HPLC separation, while the $R_{p}$ fractions are completely digested into single nucleotides. Digestion mixtures contained $2.5 \mathrm{nmol}$ RNA, $10 \mathrm{mM}$ Tris $(\mathrm{pH} \mathrm{8}), 10 \mathrm{mM} \mathrm{MgCl}_{2}, 0.1$ unit of snake venom phosphodiesterase I (Sigma-Aldrich), and 1 unit of calf intestine alkaline phosphatase (USB). Reactions were incubated for $1 \mathrm{~h}$ at $37^{\circ} \mathrm{C}$. A dinucleotide peak was observed only in fractions from the second peak, identifying this peak as the $S_{p}$ distereomer fraction.

\section{HHRz cleavage assays}

Catalytic activities of the HHRz were determined by using the 43nt enzyme strand (ES) and $5^{\prime}{ }^{32} \mathrm{P}$-labeled 20 -nt substrate strand (S). Substrate strands were radioactively labeled $\left(5^{\prime}-{ }^{32} \mathrm{P}\right)$ using $\left[\gamma-{ }^{32} \mathrm{P}\right]$ ATP and polynucleotide kinase (New England Biolabs). Labeled RNA was purified by PAGE, eluted in $100 \mathrm{mM}$ acetic acid ( $\mathrm{pH} 4.0)$ with TEMED, and desalted by EtOH precipitation and run through a G-25 column. The enzyme strand at $3 \mu \mathrm{M}$ was mixed with trace-labeled substrate, in the presence of $2 \times \mathrm{Na}^{+}$and $100 \mathrm{mM}$ Bis-tris propanediol buffer ( $\mathrm{pH}$ 6.5). The complex was annealed by heating for $2 \mathrm{~min}$ to $90^{\circ} \mathrm{C}$, cooling for $20 \mathrm{~min}$ to $4^{\circ} \mathrm{C}$, and equilibrating for $10 \mathrm{~min}$ at $20^{\circ} \mathrm{C}$. Reactions were initiated by the addition of an equal volume of $2 \times\left[\mathrm{Ca}^{2+}\right]$ and $2 \times\left[\mathrm{Cd}^{2+}\right]$ to the annealed RNA. The final conditions were $1.5 \mu \mathrm{M}$ ES, trace $\mathrm{S}^{*}$, $1 \times \mathrm{Na}, 50 \mathrm{mM}$ buffer, $1 \times \mathrm{Ca}^{2+}$, and $1 \times \mathrm{Cd}^{2+}$. Time points were quenched by the addition of $166 \mathrm{mM}$ EDTA in $66 \%$ formamide 


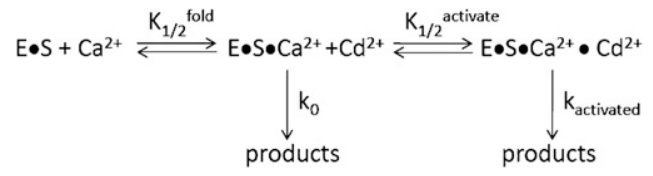

(SCHEME 1)

with trace amounts of xylenecyanol and Bromophenol Blue. Cleavage products were separated by dPAGE, then quantified using storage phosphor screens (Kodak) and imaging by Imagequant. A zero-time-point correction was used to account for background cleavage during the annealing steps.

We assume a model in which the rate in $\mathrm{Ca}^{2+}$ represents the folded, but nonactivated $\mathrm{HHRz}$, and the addition of $\mathrm{Cd}^{2+} \mathrm{ac}-$ tivates the ribozyme (Scheme 1).

Rate constants were determined by fitting the corrected fraction cleaved versus time to either a monophasic (Eq. 1) or biphasic (Eq. 2) model in which A and B represent the extents of cleavage and $k_{\text {obs1 }}$ and $k_{\text {obs2 }}$ are observed rate constants (Kim et al. 2005; Osborne et al. 2005).

$$
\begin{gathered}
y(t)=A\left(1-e^{-k_{o b s 1}^{t}}\right) \\
y(t)=A\left(1-e^{-k_{o b s 1}^{t}}\right)+B\left(1-e^{-k_{o b s 2}^{t}}\right)
\end{gathered}
$$

Most fits of "native" HHRz kinetic data are best described by a model of two noninteracting populations, with the faster phase corresponding to the majority population and a slow phase of $<0.01 \mathrm{~min}^{-1}$. In the case of the $\mathrm{R}_{\mathrm{p}}-\mathrm{S}$ substitution, Equation $1 \mathrm{fit}$ the very slow rates in 0 to $\sim 10^{-6} \mathrm{M}\left[\mathrm{Cd}^{2+}\right]$, and Equation 2 was used when the fitting parameters indicated $\sim 50 \%$ for the second population and significantly improved values of $R^{2}$. The $\mathrm{Cd}^{2+}$ dependence of the rate constant for the fastest population was fit to a binding equation (3):

$$
k_{\text {activated }}=k_{0}+\frac{k_{\max } x^{n}}{k_{1 / 2}^{n}+x^{n}}
$$

where $k_{0}$ is set as the rate in only $\mathrm{Na}^{+}$and $\mathrm{Ca}^{2+}, k_{\text {max }}$ is the predicted maximum rate of cleavage at saturating $\mathrm{Cd}^{2+}$, and $n$ is the cooperativity coefficient. Nonlinear regression fits were performed using SigmaPlot 11 (Systat Software).

\section{ACKNOWLEDGMENTS}

We are grateful for financial support from the NIH (GM058096 to V.J.D. and GM007759-29 to W.L.W.) and the NSF (DGE-0742540 to W.L.W.).

Received September 2, 2011; accepted October 11, 2011.

\section{REFERENCES}

Bevilacqua PC. 2003. Mechanistic considerations for general acid-base catalysis by RNA: Revisiting the mechanism of the hairpin ribozyme. Biochemistry 42: 2259-2265.
Bevilacqua PC, Brown TS, Nakano S, Yajima R. 2004. Catalytic roles for proton transfer and protonation in ribozymes. Biopolymers 73: 90-109.

Blount KF, Uhlenbeck OC. 2005. The structure-function dilemma of the hammerhead ribozyme. Annu Rev Biophys Biomol Struct 34: $415-440$.

Boots JL, Canny MD, Azimi E, Pardi A. 2008. Metal ion specificities for folding and cleavage activity in the Schistosoma hammerhead ribozyme. RNA 14: 2212-2222.

Buzayan JM, Gerlach WL, Bruening G. 1986. Satellite tobacco ringspot virus RNA: A subset of the RNA sequence is sufficient for autolytic processing. Proc Natl Acad Sci 83: 8859-8862.

Chi YI, Martick M, Lares M, Kim R, Scott WG, Kim SH. 2008. Capturing hammerhead ribozyme structures in action by modulating general base catalysis. PLoS Biol 6: e234. doi: 10.1371/ journal.pbio.0060234.

de la Pena M, Garcia-Robles I. 2010. Ubiquitous presence of the hammerhead ribozyme motif along the tree of life. RNA 16: 19431950.

DeRose VJ. 2003. Metal ion binding to catalytic RNA molecules. Curr Opin Struct Biol 13: 317-324.

Erat MC, Roland KO. 2011. Methods to detect and characterize metal ion binding sites in RNA. Met Ions Life Sci 9: 37-100.

Forconi M, Herschlag D. 2009. Use of phosphorothioates to identify sites of metal-ion binding in RNA. Methods Enzymol 468: 311-333.

Frederiksen JK, Piccirilli JA. 2009. Identification of catalytic metal ion ligands in ribozymes. Methods 49: 148-166.

Horton TE, Clardy DR, DeRose VJ. 1998. Electron paramagnetic resonance spectroscopic measurement of $\mathrm{Mn}^{2+}$ binding affinities to the hammerhead ribozyme and correlation with cleavage activity. Biochemistry 37: 18094-18101.

Hunsicker LM, DeRose VJ. 2000. Activities and relative affinities of divalent metals in unmodified and phosphorothioate-substituted hammerhead ribozymes. J Inorg Biochem 80: 271-281.

Khvorova A, Lescoute A, Westhof E, Jayasena SD. 2003. Sequence elements outside the hammerhead ribozyme catalytic core enable intracellular activity. Nat Struct Biol 10: 708-712.

Kim NK, Murali A, DeRose VJ. 2005. Separate metal requirements for loop interactions and catalysis in the extended hammerhead ribozyme. J Am Chem Soc 127: 14134-14135.

Lee TS, Silva-López C, Martick M, Scott WG, York DM. 2007. Insight into the role of $\mathrm{Mg}^{2+}$ in hammerhead ribozyme catalysis from $\mathrm{X}$-ray crystallography and molecular dynamics simulation. J Chem Theory Comput 3: 325-327.

Lee TS, López CS, Giambasu GM, Martick M, Scott WG, York DM. 2008. Role of $\mathrm{Mg}^{2+}$ in hammerhead ribozyme catalysis from molecular simulation. J Am Chem Soc 130: 3053-3064.

Lee TS, Giambasu GM, Sosa CP, Martick M, Scott WG, York DM. 2009. Threshold occupancy and specific cation binding modes in the hammerhead ribozyme active site are required for active conformation. J Mol Biol 388: 195-206.

Maderia M, Hunsicker LM, DeRose VJ. 2000. Metal-phosphate interactions in the hammerhead ribozyme observed by ${ }^{31} \mathrm{P}$ NMR and phosphorothioate substitutions. Biochemistry 39: 1211312120.

Martick M, Scott WG. 2006. Tertiary contacts distant from the active site prime a ribozyme for catalysis. Cell 126: 309-320.

Martick M, Lee T-S, York DM, Scott WG. 2008. Solvent structure and hammerhead ribozyme catalysis. Chem Biol 15: 332-342.

Murray JB, Seyhan AA, Walter NG, Burke JM, Scott WG. 1998. The hammerhead, hairpin and VS ribozymes are catalytically proficient in monovalent cations alone. Chem Biol 5: 587-595.

Nesbitt S, Hegg LA, Fedor MJ. 1997. An unusual pH-independent and metal-ion-independent mechanism for hairpin ribozyme catalysis. Chem Biol 4: 619-630.

O'Rear JL, Wang SL, Feig AL, Beigelman L, Uhlenbeck OC, Herschlag D. 2001. Comparison of the hammerhead cleavage reactions stimulated by monovalent and divalent cations. RNA 7: 537-545. 
Osborne EM, Schaak JE, DeRose VJ. 2005. Characterization of a native hammerhead ribozyme derived from schistosomes. RNA 11: 187196.

Osborne EM, Ward WL, Ruehle MZ, DeRose VJ. 2009. The identity of the nucleophile substitution may influence metal interactions with the cleavage site of the minimal hammerhead ribozyme. Biochemistry 48: 10654-10664.

Pecoraro VL, Hermes JD, Cleland WW. 1984. Stability constants of $\mathrm{Mg}^{2+}$ and $\mathrm{Cd}^{2+}$ complexes of adenine nucleotides and thionucleotides and rate constants for formation and dissociation of MgATP and MgADP. Biochemistry 23: 5262-5271.

Peracchi A, Beigelman L, Scott EC, Uhlenbeck OC, Herschlag D. 1997. Involvement of a specific metal ion in the transition of the hammerhead ribozyme to its catalytic conformation. J Biol Chem 272: 26822-26826.

Pley HW, Flaherty KM, McKay DB. 1994. Three-dimensional structure of a hammerhead ribozyme. Nature 372: 68-74.

Scott WG, Finch JT, Klug A. 1995. The crystal structure of an all-RNA hammerhead ribozyme: A proposed mechanism for RNA catalytic cleavage. Cell 81: 991-1002.

Sigel RK, Pyle AM. 2007. Alternative roles for metal ions in enzyme catalysis and the implications for ribozyme chemistry. Chem Rev 107: 97-113.
Sigel RKO, Song B, Sigel H. 1997. Stabilities and structures of metal ion complexes of adenosine $5^{\prime}$-O-thiomonophosphate (AMPS ${ }^{2-}$ ) in comparison with those of its parent nucleotide $\left(\mathrm{AMP}^{2-}\right)$ in aqueous solution. J Am Chem Soc 119: 744-755.

Slim G, Gait MJ. 1991. Configurationally defined phosphorothioatecontaining oligoribonucleotides in the study of the mechanism of cleavage of hammerhead ribozymes. Nucleic Acids Res 19: 11831188.

Steitz TA, Steitz JA. 1993. A general two-metal-ion mechanism for catalytic RNA. Proc Natl Acad Sci 90: 6498-6502.

Suzumura K, Yoshinari K, Tanaka Y, Takagi Y, Kasai Y, Warashina M, Kuwabara T, Orita M, Taira K. 2002. A reappraisal, based on ${ }^{31} \mathrm{P}$ NMR, of the direct coordination of a metal ion with the phosphoryl oxygen at the cleavage site of a hammerhead ribozyme. J Am Chem Soc 124: 8230-8236.

Vogt M, Lahiri S, Hoogstraten CG, Britt RD, DeRose VJ. 2006. Coordination environment of a site-bound metal ion in the hammerhead ribozyme determined by ${ }^{15} \mathrm{~N}$ and ${ }^{2} \mathrm{H}$ ESEEM spectroscopy. J Am Chem Soc 128: 16764-16770.

Wang SL, Karbstein K, Peracchi A, Beigelman L, Herschlag D. 1999. Identification of the hammerhead ribozyme metal ion binding site responsible for rescue of the deleterious effect of a cleavage site phosphorothioate. Biochemistry 38: 14363-14378. 

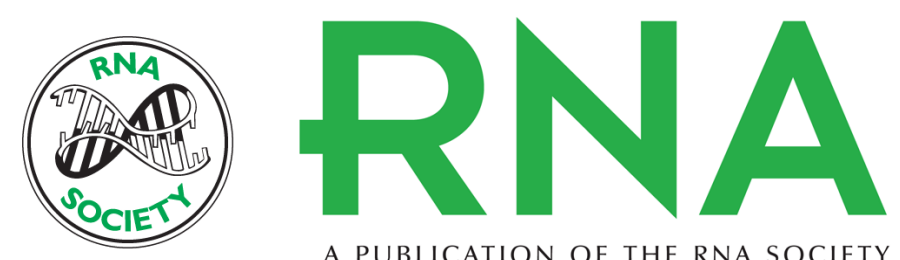

A PUBLICATION OF THE RNA SOCIETY

\section{Ground-state coordination of a catalytic metal to the scissile phosphate of a tertiary-stabilized Hammerhead ribozyme}

W. Luke Ward and Victoria J. DeRose

RNA 2012 18: 16-23 originally published online November 28, 2011

Access the most recent version at doi:10.1261/rna.030239.111

\section{References This article cites 37 articles, 7 of which can be accessed free at: http://rnajournal.cshlp.org/content/18/1/16.full.html\#ref-list-1}

\section{License}
Email Alerting Receive free email alerts when new articles cite this article - sign up in the box at the Service top right corner of the article or click here.

